\title{
Clinical diagnosis, treatment and screening of the VHL gene in three von Hippel-Lindau disease pedigrees
}

\author{
GUOBING LIN $^{1}$, YIHUA ZHAO ${ }^{2}$, ZHEWEI ZHANG $^{3}$ and HUIJIANG ZHANG ${ }^{4}$ \\ ${ }^{1}$ Department of Urology, The First People's Hospital of Wenling, Wenling, Zhejiang 317500; \\ ${ }^{2}$ Department of Urology, Yueqing People's Hospital, Yueqing, Zhejiang 325600; ${ }^{3}$ Department of Urology, \\ The Second Affiliated Hospital of Zhejiang University School of Medicine, Hangzhou, Zhejiang 310009; \\ ${ }^{4}$ Department of Urology, Lishui City People's Hospital, Lishui, Zhejiang 323000 P.R. China
}

Received September 1, 2018; Accepted August 23, 2019

DOI: $10.3892 / \mathrm{etm} .2020 .8829$

\begin{abstract}
The present study aimed to investigate the clinical characteristics of von Hippel-Lindau (VHL) disease and the clinical significance of VHL gene detection. The clinical materials of patients with VHL disease were collected from 3 different families between May 1985 and October 2017. A systematic pedigree study and VHL gene detection at the germline level were performed together with a literature review. Of the 22 patients from 3 VHL pedigrees, 10 exhibited VHL gene mutations (3 genotypes) at the germline level. The genotypes of pedigree were VHL-p.R161Q (c.482G>A), VHL-p.N78S (c.233A>G), and VHL-p.R167Q (c.500G>A). During the follow-up period, the symptoms were stable in 10 patients, including 2 cases of central nervous system hemangioblastomas (CNS-HB), 3 cases of bilateral multiple renal cell carcinoma (RCC) and 5 cases of adrenal pheochromocytoma without local recurrence or distant metastasis. Patients with p.R161Q and p.N78S were not associated with CNS-HB, which was different from the clinical phenotype of previously reported families. RCC were Fuhrman II grade, which was consistent with the previous study. The results of the present study indicated that the standardization of early diagnosis and the improvement of long-term efficacy may be achieved by combining clinical screening and VHL gene detection.
\end{abstract}

\section{Introduction}

Von Hippel-Lindau (VHL) disease (1) is a rare autosomal dominant inherited disease that predisposes the affected individual to various benign or malignant tumors with an incidence rate of 1 in every 36,000-50,000 worldwide in

Correspondence to: Dr Huijiang Zhang, Department of Urology, Lishui City People's Hospital, 15 Dazhong Street, Lishui, Zhejiang 323000, P.R. China

E-mail: iszhanghuijiang@163.com

Key words: von Hippel-Lindau disease, VHL gene, molecular diagnosis, case report
2018 (2-4). Currently, >40 lesions for VHL disease have been primarily observed in the central nervous system (CNS) and in 14 different organs, including hemangioblastomas (HB; $44-72 \%$ of cases), renal cell carcinoma (RCC; $25-45 \%$ of cases), pheochromocytoma (PHEO)/paragangliomas (PGL; $10-30 \%$ of cases), endolymphatic sac tumors (ELSTs; $6-15 \%$ of cases), renal cysts (60\% of cases), pancreatic neuroendocrine tumors (PNETs; 5-10\% of cases), pancreatic serous cystadenoma/cysts (72-90\% of cases), and papillary cystadenoma (PC) of the epididymis and the broad ligament of the uterus (2-15). VHL disease is primarily caused by inactivation of the VHL tumor-suppressive protein. The VHL gene (OMIM, 608537) is located on human chromosome 3p25.3 and encodes the VHL protein (pVHL), which forms a complex with elongation factor and is critical for pVHL to function as an E3 ligase (16). VHL germline mutations result in the dysfunction of E3 ligase and the accumulation of hypoxia-inducible factor- $\alpha$ (HIF- $\alpha$; VHL-Elongin-HIF- $\alpha$ complex), leading to decreased ubiquitylation and proteasomal degradation of HIFs, namely HIF-1 $\alpha$ and HIF-2 $\alpha$. Elevated levels of HIFs subsequently regulate overactivation of the downstream pathways in which vascular endothelial growth factor (VEGF), platelet-derived growth factor- $\beta$ (PDGF- $\beta$ ) and transforming growth factor- $\alpha$ (TGF- $\alpha$ ) are involved, which accelerates tumorigenesis (14). The present study analyzed the clinical data of 9 patients from 3 families with VHL disease. The mutations in the VHL gene of patients and their family members were determined.

\section{Materials and methods}

Patients. Between May 1985 and October 2017, three Han pedigrees with VHL disease were recruited separately and followed up by Lishui People's Hospital (Lishui, China), the Second Affiliated Hospital of Zhejiang University School of Medicine (Hangzhou, China) and the First People's Hospital of Wenling City (Wenling, China), respectively. The patients underwent collection of their medical history, family survey (pedigree analysis) and a detailed physical examination and associated auxiliary examination by a specialist (GL, ZZ and HZ, respectively) from each of the three hospitals aforementioned in a blinded manner. There were 21 members in the pedigrees, including 15 males and 6 females, aged 9-66 years (Fig. 1 and Table I). The present study was 


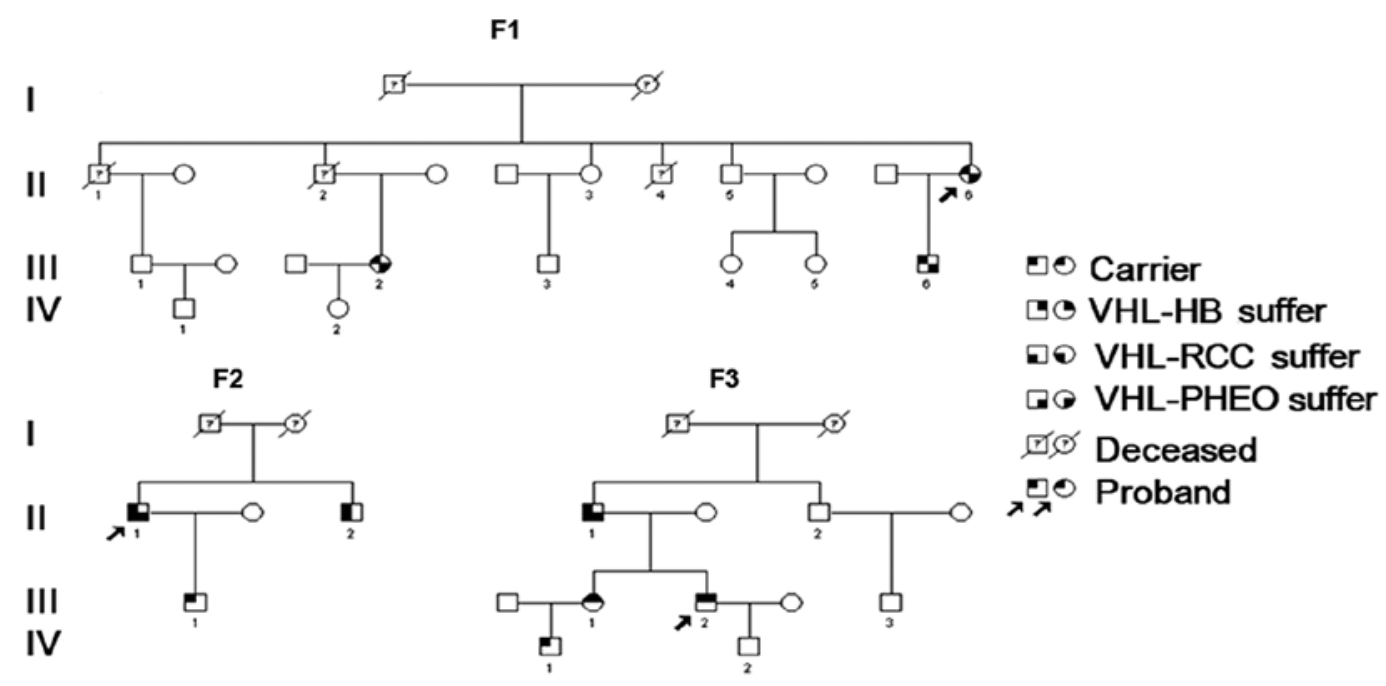

Figure 1. Pedigree tree of three families with VHL disease. F1, family 1; F2, family 2; F3, family 3; Squares, male; circles, female. VHL, von Hippel-Lindau; HB, hemangioblastoma; RCC, renal cell carcinoma; PHEO, pheochromocytoma.

approved by the Ethics Committee of Lishui People's Hospital and written informed consent was obtained from all patients.

Detection of VHL gene mutation. A total of $5 \mathrm{ml}$ peripheral blood (EDTA anticoagulant) was collected from each of the 21 family members from the 3 families, including the 3 probands (F1-II6, F2-II1 and F3-III2). Extraction of genomic DNA from peripheral blood leukocytes was performed using a QIAamp Blood kit (Qiagen $\mathrm{GmbH}$ ) according to the manufacturer's protocol. The primer sequences were designed based on the VHL gene sequence found in GeneBank using the Primer-Blast online tool (https://omictools.com/primer-blast-tool) and synthesized by Sangon Biotech Co., Ltd. The primer sequences were forward, 5'-ACCGGTGTGGCTCTTTAACA-3' and reverse, 5'-TCCTGTACTTACCACAACAACCTT-3'. The $20 \mu \mathrm{l}$ solution used for the PCR amplification consisted of $17 \mu 1$ KAPA2G Robust HotStart ReadyMix (cat. no. KK5701; Beijing Huaruikang Technology Co., Ltd.), $1 \mu \mathrm{l}$ each of the aforementioned upstream and downstream primers $(10 \mu \mathrm{M})$ and $1 \mu \mathrm{l}$ of genomic DNA (200 ng) from both the patients and family members. PCR was performed as follows: Pre-denaturation at $94^{\circ} \mathrm{C}$ for $5 \mathrm{~min}$, followed by 30 cycles of denaturation at $94^{\circ} \mathrm{C}$ for $30 \mathrm{sec}$, annealing at $65^{\circ} \mathrm{C}$ for $30 \mathrm{sec}, 72^{\circ} \mathrm{C}$ for $1 \mathrm{~min}$; and extension at $72^{\circ} \mathrm{C}$ for $5 \mathrm{~min}$. The $2 \mu \mathrm{lPCR}$ amplified product was used for subsequent electrophoresis with $1 \%$ agarose gel for identification and purification. The gel was stained with ethidium bromide (cat. no. E7637; Sigma-Aldrich; Merck KGaA) for visualization. The purified product was sent to Shanghai Xiang Yin Biotechnology Co.,Ltd. for sequencing with an ABI 3730XL sequencer. All mutations were confirmed by bi-directional sequencing.

Imaging examination and detection of biochemical indexes. The brain, adrenal glands, kidney, and pancreas were imaged using ultrasound, CT or MRI scans and fundus screening was performed in patients with VHL mutations. Serological tests, including the measurement of catecholamine and/or urine vanillicmandelic acid for $24 \mathrm{~h}$, were performed using an AU400 Automated Chemistry Analyzer (Olympus Corporation).
Follow-up. All patients were followed up by telephone or hospital visits for 5 years. The follow-up interval was every 2 months in the first year, then every 6 months in the second year and once every year thereafter. During follow-up, general conditions, including blood pressure, blood biochemical indices, recurrence or metastasis of tumor were assessed for every patient.

\section{Results}

Family 1 (F1) investigation and general clinical material. Proband (F1-II6) was female and aged 50 years. The patient was admitted to Lishui People's Hospital (Lishui, China) in May 1985 due to paroxysmal headache, palpitation and hyperhidrosis that had occurred for 3 months. The results of physical examinations revealed that patient heart rate was $110 \mathrm{bpm}$ and blood pressure was $210 / 120 \mathrm{mmHg}$. Ultrasound and CT results revealed a mass sized $5.5 \times 4.5 \times 2.8 \mathrm{~cm}$ on the right side of the adrenal gland and it was considered as right PHEO. Retinal hemangioblastoma was excluded by an ophthalmologist. After blood pressure and volume expansion were adjusted with phenylbenzylamine hydrochloride tablets, right adrenal tumor resection was performed under general anesthesia. The postoperative pathology results confirmed that the patient suffered from right PHEO.

The proband's son (F1-III6) was 25 years old and was admitted to Lishui People's Hospital (Lishui, China) in March 2002 due to paroxysmal headache and hypertension (140-160/100-110 mmHg). The results of the physical examination revealed that his levels of serum catecholamine were increased, including dopamine $(88.64 \mathrm{ng} / \mathrm{l}$; normal reference value, $<100 \mathrm{ng} / \mathrm{l})$, adrenaline (106.23 ng/l; normal reference value $<600 \mathrm{ng} / \mathrm{l})$ and norepinephrine $(827.15 \mathrm{ng} / \mathrm{l}$; normal reference value $<100 \mathrm{ng} / \mathrm{l})$. Ultrasound and CT results revealed a mass sized $5.6 \times 3.8 \times 3.0 \mathrm{~cm}$ on the right side of the adrenal gland. After blood pressure and volume expansion were adjusted with phenylbenzylamine hydrochloride tablets, right adrenal tumor laparoscopic resection was performed under general anesthesia. The postoperative pathology results confirmed that the 


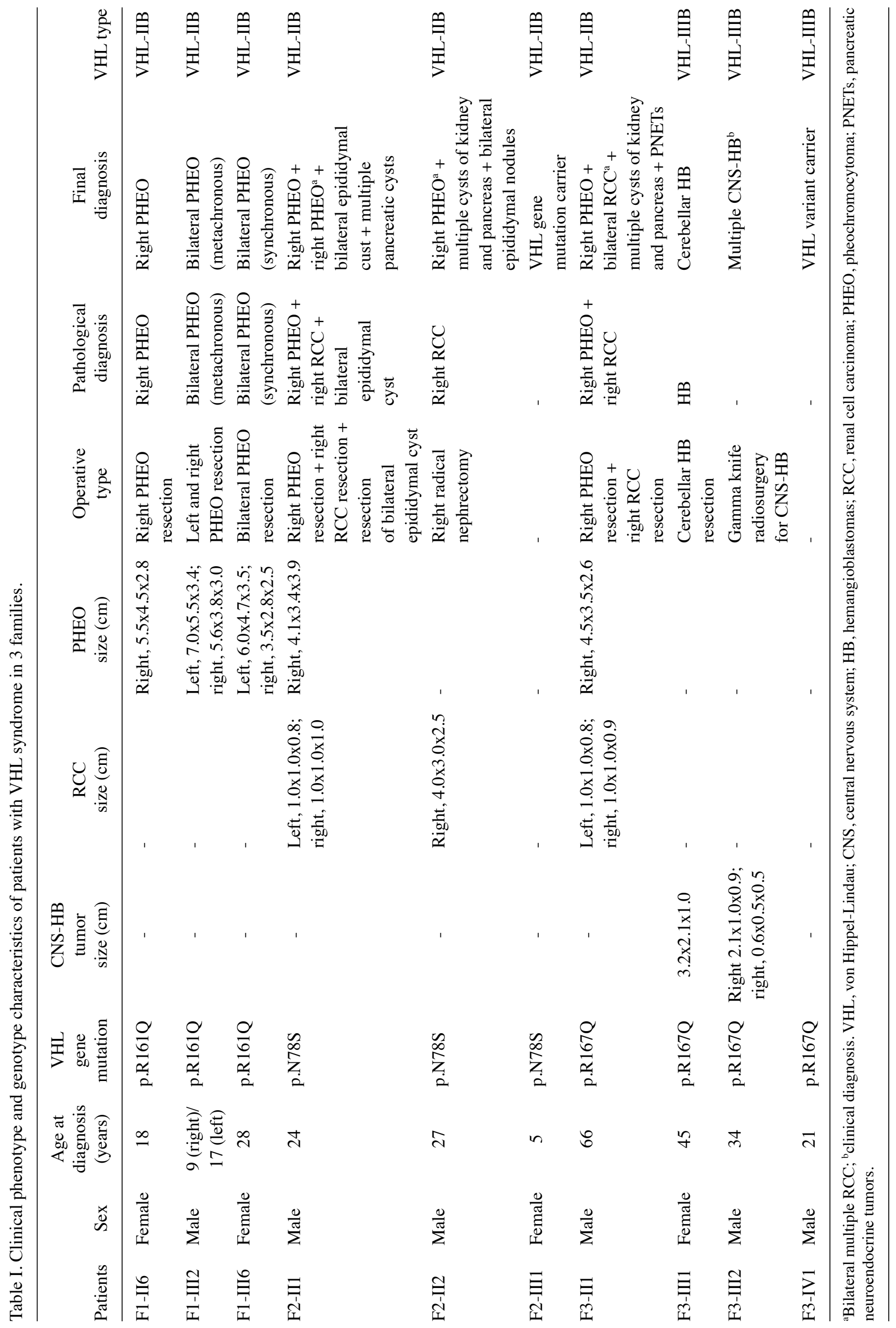


A

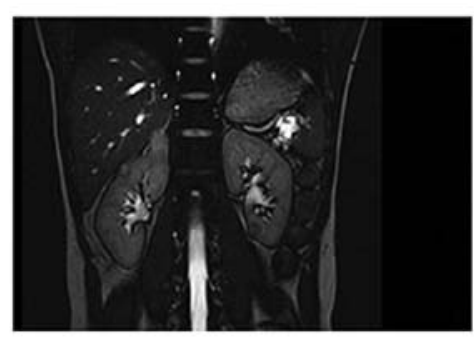

D

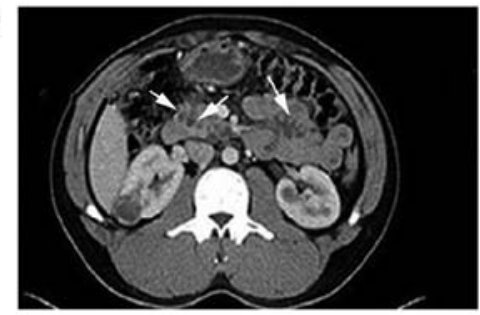

B
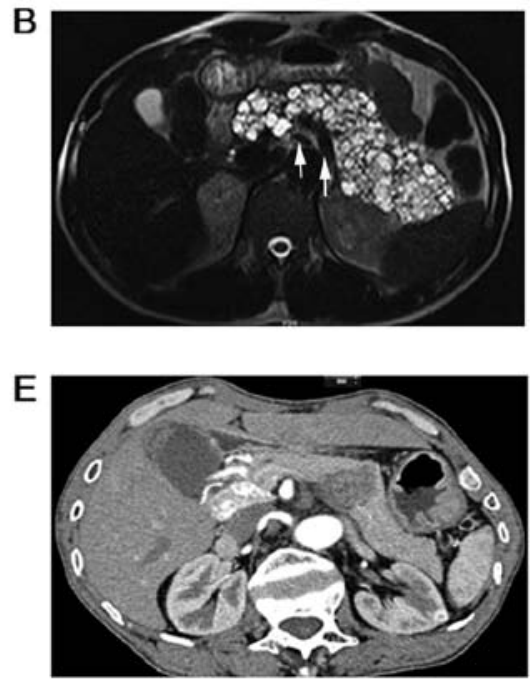

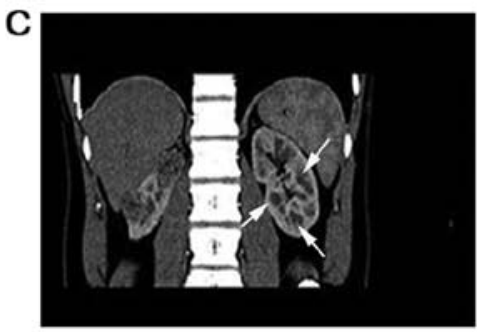

$\mathrm{F}$

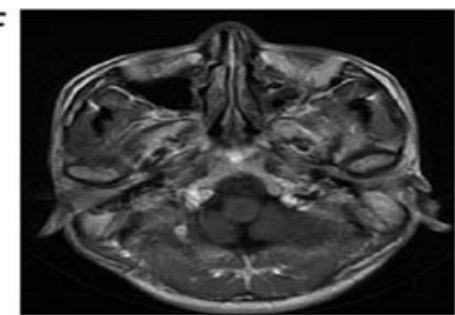

Figure 2. Imaging examination results. (A) Coronal and (B) axial abdominal MRI revealed right PHEO, multiple renal RCC and multiple pancreatic cysts in F2-II1. Arrows indicate cysts. (C) Coronal and (D) axial abdominal CT revealed multiple RCC of the right kidney with cystic degeneration and multiple pancreatic cysts in F2-II2. Arrows indicate cysts. (E) Axial abdominal CT revealed right PHEO, left RCC and neuroendocrine tumors of pancreatic head in F3-II1. (F) Axial cranial MRI revealed hemangioblastoma with a clear boundary. The right margin of the sellar region and vermis of cerebellum were significantly enhanced in F3-III2. PHEO, pheochromocytoma; RCC, renal cell carcinoma; F, family.

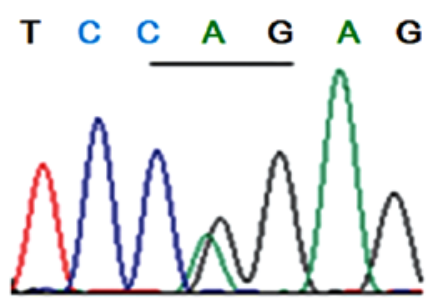

A
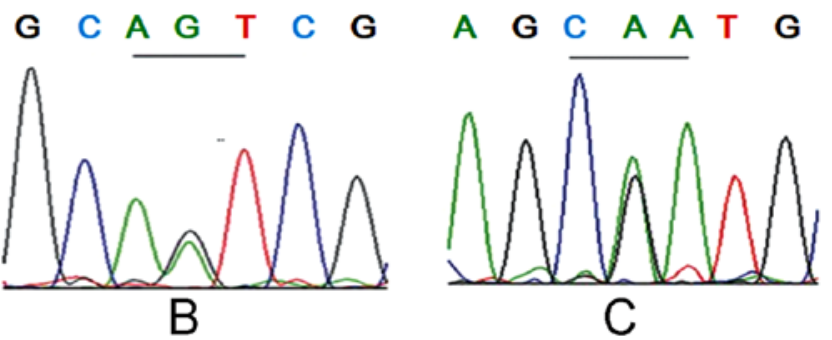

Figure 3. Results of VHL gene detection. (A) A G to A heterozygous mutation (p.R161Q) was presented in the 482nd codon of proband F1-II11. (B) An A to G missense mutation (p.N78S) in the 233rd codon of the proband F2-II1 was identified and (C) a G to A heterozygous mutation (p.R167Q) in the 500th codon was exhibited in the proband F3-III1. VHL, von Hippel-Lindau; F, family.

patient suffered from right PHEO. In 2010, this patient suffered from paroxysmal hypertension and headache for a second time and his levels of serum catecholamine were also increased, including dopamine $(600.36 \mathrm{ng} / \mathrm{l})$, adrenaline (297.94 ng/l) and norepinephrine $(1,093.84 \mathrm{ng} / \mathrm{l})$. The ultrasound and CT results revealed a mass sized $7.0 \times 5.5 \times 3.4 \mathrm{~cm}$ on the left side of the adrenal gland. After blood pressure and volume expansion were adjusted with phenylbenzylamine hydrochloride tablets, left adrenal tumor resection was performed under general anesthesia. The postoperative pathology results confirmed that the patient suffered from left PHEO. The final diagnosis of this patient was bilateral PHEO.

The proband's niece (F1-III2) was 38 years old and was admitted to Lishui People's Hospital in October 2002 due to persistent headache and dizziness that had lasted for 3 days. The results of physical examination demonstrated that the patient's blood pressure was $160 / 100 \mathrm{mmHg}$ and their serum catecholamine levels were increased. Ultrasound/CT revealed a bilateral mass on the adrenal gland. The left mass was $6.0 \times 4.7 \times 3.5 \mathrm{~cm}$ in size and the right mass was $3.5 \times 2.8 \times 2.5 \mathrm{~cm}$ in size. After blood pressure and volume expansion were adjusted with phenylbenzylamine hydrochloride tablets, bilateral adrenal tumor laparoscopic resection was performed under general anesthesia. The postoperative pathology results confirmed that the patient suffered from bilateral PHEO. The father of this patient (F1-II2, the proband's brother) died from hypertensive intracerebral hemorrhage 26 years ago, aged 38 years.

Family 2 (F2) investigation and general clinical material. The proband (F2-II1) of F2 was male and aged 33 years. The patient was admitted to the Second Affiliated Hospital of Zhejiang University School of Medicine (Hangzhou, China) in September 2009 due to paroxysmal headache, dizziness and palpitation with hyperhidrosis. Physical examination results revealed that the patients' heart rate was $100 \mathrm{bpm}$ and blood pressure was $105 / 78 \mathrm{mmHg}$. MRI results revealed a mass sized $4.1 \times 3.4 \times 3.9 \mathrm{~cm}$ at the right adrenal gland and multiple renal tumors (the largest diameter of the tumors, $1.0 \times 1.0 \times 1.0 \mathrm{~cm}$ on the left; $1.0 \times 1.0 \times 0.8 \mathrm{~cm}$ on the right) and multiple cysts in the pancreas (Fig. 2A and B). An ophthalmologist (from the Second Affiliated Hospital of Zhejiang University School of Medicine) excluded the existence of retinal hemangioblastoma preoperatively. The concentration of urine vanillicmandelic acid at $24 \mathrm{~h}$ was $138.3 \mu \mathrm{mol} / 24 \mathrm{~h}$ (normal reference value, $<33 \mu \mathrm{mol} / \mathrm{d}$ ). Combined with clinical 
manifestations, the patient was considered as right PHEO, with double renal masses and multiple pancreatic cysts. After the patient's blood pressure and volume expansion was adjusted with phenylbenzylamine hydrochloride tablets, resection of the right adrenal tumor and the upper pole of the right kidney were performed under general anesthesia. The postoperative pathology results confirmed that the patient had right PHEO and right RCC (Fuhrman II grade) (17). In April 2012, the patient underwent bilateral epididymal nodule excision due to the presence of multiple nodules in the bilateral epididymal head. Postoperative pathology results confirmed that the patient suffered from papillary cystadenoma of the bilateral epididymal head.

The proband's younger brother (F2-II2) was aged 32 years. In 2012, the patient presented with multiple masses in both kidneys (left, $1.0 \times 1.0 \times 1.0 \mathrm{~cm}$; right, $4.0 \times 3.5 \times 2.6 \mathrm{~cm}$ ), multiple cysts in both kidneys, and pancreas and epididymal nodules (Fig. 2C and D). The patient underwent laparoscopic right radical nephrectomy under general anesthesia. The postoperative pathology results confirmed that the patient suffered from multiple RCC in the right kidney (Fuhrman II grade). IFN and IL-2 were used for postoperative treatment.

Family 3 (F3) investigation and general clinical material. Proband (F3-III2) was male and aged 39 years. In April 2013, the patient was admitted to the First People's Hospital of Wenling City (Wenling, China) having suffered with dizziness and headaches for 1 month. A cranial MRI examination revealed that there was a mass sized $2.1 \times 1.0 \times 0.9 \mathrm{~cm}$ at the right margin of the sellar region and a mass sized $0.6 \times 0.5 \times 0.5 \mathrm{~cm}$ at the cerebellar vermis. The masses were markedly enhanced with clear margins. The patient was considered to suffer from CNS-HB. An ophthalmologist (The First People's Hospital of Wenling City) excluded retinal hemangioblastoma. As the patient refused surgical treatment, cranial gamma knife radiotherapy (peripheral dose $5 \mathrm{~Gy}$, central dose $10 \mathrm{~Gy}$ ) was performed 6 times with a 5 Gy dose at the peripheral and 10 Gy dose at the central positions. CNS-HB of the F3-III2 was stable after 5 years of follow-up.

The proband's elder sister was aged 45 years (F3-III1). In May 2015, the patient was admitted to the First People's Hospital of Wenling City due to dizziness lasting 3 months. A brain MRI revealed that there was a mass sized $3.2 \times 2.1 \times 1.0 \mathrm{~cm}$ at the vermis of the cerebellum, with clear margins. The mass was excised via surgery. The postoperative pathology results confirmed that the patient suffered from cerebellar hemangioblastoma.

The proband's father (F3-II1) was aged 66 years. The patient was hospitalized in the First People's Hospital of Wenling City presenting with cough and expectoration with low fever for 2 months in February 2014. Physical examination results revealed that the patient's temperature was $37.8^{\circ} \mathrm{C}$ and his blood pressure fluctuated between $104-123 / 66-96 \mathrm{mmHg}$. CT examination results revealed that the patient suffered from chronic obstructive pulmonary disease with pulmonary infection. Ultrasound and CT results revealed a mass sized $4.5 \times 3.5 \times 2.6 \mathrm{~cm}$ on the right side of the adrenal gland, multiple solid lesions of both kidneys (left, $1.0 \times 1.0 \times 0.8 \mathrm{~cm}$; right, $1.0 \times 1.0 \times 0.9 \mathrm{~cm}$ ) with multiple small cysts and pancreatic head lesions $(2.6 \times 2.0 \times 2.0 \mathrm{~cm})$. Following treatment of a lung infection and the adjustment of blood pressure and volume expansion with phenylbenzylamine hydrochloride tablets, resection of the right adrenal tumor and right kidney tumor were performed under general anesthesia. The postoperative pathology results revealed that the patient suffered from right PHEO and right RCC (Fuhrman II grade).

VHL gene detection results. Of the 22 patients from 3 VHL families, 10 had three VHL germline missense mutations within coding regions, respectively (Fig. 3). In the F1 family, 3 cases harbored the p.R161Q (c.482G $>$ A) mutation at exon 3 (Fig. 3A); 3 cases in the F2 family harbored the p.N78S (c.233A $>$ G) mutation at exon 1 (Fig. 3B); and 4 cases in the F4 family harbored the p.R167Q (c.500G $>$ A) mutation at exon 3 (Fig. 3C). Apart from 8 patients with the VHL clinical phenotype who had been surgically and pathologically diagnosed, 2 new cases (F2-III1 and F3-IV2) were revealed to be asymptomatic carriers of VHL gene mutations. The remaining 12 family members had no VHL gene mutations and no clinical manifestations or abnormalities following imaging and serological tests associated with VHL disease. The clinical phenotype and genotype characteristics are presented in Table I.

\section{Discussion}

VHL disease, also known as VHL syndrome, is characterized by a variety of benign, malignant tumors and multiple organ cysts (18). VHL patients usually develop clinical symptoms after reaching 20 years of age, with $90-100 \%$ penetrance of clinical symptoms between 65 and 70 years old (19). Retinal and cerebellar HB are typically the most common and earliest presenting forms of VHL disease $(4,7,9)$. VHL syndrome can be divided into two types: Type I and type II. Type I syndrome has CNS-HB and/or RCC among other tumors (except PHEO) and can also be divided into type IA (high-risk RCC) and type IB (without RCC). The patients with type II must have PHEO and can be divided into type IIA (includes PHEO but not RCC), type IIB (RCC + PHEO) and type IIC (only PHEO) $(2,3,14)$. The clinical diagnosis of this disease should be based on VHL symptoms, heredity and family factors. If a patient possesses one of the following three conditions, they may be diagnosed with VHL disease: i) At least two positions where CNS-HB exists; ii) CNS-HB at one position and one other organ tumor (RCC, PHEO/PGL, PNETs or ELSTs); iii) at least one visceral tumor (RCC, PHEO/PGL, PNETs or ELSTs) associated with the VHL gene mutation at the pathogenic germline level, or a parent has been diagnosed with VHL (3). Despite the high heterogeneity of clinical phenotypes, VHL gene mutations could be detected at the germline level in almost all patients with VHL disease (2). The patients in the three families included in the present study were diagnosed as VHL type II. The patients in F2 and F3 were VHL type IIB and the patients in F1 were VHL type IIC (Table I).

The VHL gene contains three exons and encodes a polysaccharide anchored membrane protein containing 213 amino acids (20). The $\mathrm{N}$-terminal region of the protein contains $\alpha$ and $\beta$ domains (20). The $\alpha$ domain binds to the elongation factor $\mathrm{C} / \mathrm{B}$ to form a complex and the $\beta$ domain binds to the HIF- $\alpha$ gene (21). pVHL downregulates the expression of 
HIF- $\alpha$, activates the ubiquitination of epidermal growth factor receptor (EGFR), regulates glucose metabolism-associated genes [glucose transporter 1 (GLUT1) and posphofructokinase 1], growth factors (TGF, PDGF and VEGF) and the cascade reaction of nerve growth factor/JunB/Egl nine homolog 3-associated apoptotic pathway, ultimately leading to the formation of benign and malignant multiple organ diseases (21). Emerging evidence has indicated that HIF-1 $\alpha$ serves an important role during clear cell (cc)RCC and HB development (21). Previously, almost 400 VHL gene mutants have been revealed to cause VHL disease (22). The majority of mutations are missense (27-38\%), nonsense (13-27\%), large fragment deletions (9-20\%), microdeletions $(10 \%)$, truncation and rearrangement $(25 \%)$ mutations (22). Splicing site mutations are rare. Of all the mutations, $\sim 20 \%$ of the patients exhibited new mutations (de novo) or their parents are chimeras (22). VHL type I disease is caused primarily by a large fragment deletion of the VHL gene, including C3orf10 gene inactivation, truncation and missense mutations, which lead to the loss of pVHL function or structural changes in the protein (3). VHL type II disease associated with PHEO is mostly caused by missense mutations (78-96\%), which often lead to partial functional defects of the pVHL. The most frequent mutations of VHL type II disease are p.R167W and p.R167Q mutations at position 167 of exon 3 in the VHL gene $(2,3)$. Notably, although the stabilization of HIF- $\alpha$ is closely associated with the occurrence of RCC, both IIA and IIB antimutagenesis can inactivate VHL function, which in turn inhibits the regulation of HIF- $\alpha$ activation. However, only patients in stage IIB exhibit ccRCC (23). Currently, certain hypotheses suggest that the occurrence of ccRCC is associated with the expression of HIF-2 $\alpha(20,21)$. However, IIA mutations do not form enough HIF- $2 \alpha$ to form HB, despite producing the appropriate quantity of HIF- $\alpha$. This explains why HB is the most common clinical manifestation of VHL (21). Liu et al (15) demonstrated that patients with non-HIF- $\alpha$ binding sites demonstrated an improved survival rate when compared with those that exhibited HIF- $\alpha$ binding sites and truncated mutations (15). All patients in the three families of the present study were VHL type II with missense mutations in the VHL gene and the patients of the F3 family harbored p.R167Q $(\alpha)$ mutations accompanied with CNS-HB. No CNS-HB was observed in the F1 (p.R161Q, $\alpha$ ) and F2 (p.N78S, $\beta$ ) families, which differed from the clinical phenotype of previously reported pedigrees $(7,8)$. The presence of different clinical phenotypes or disease progression in different or identical pedigrees or members indicated that there may be other pathogenic or modifier factors, and/or secondary mutation attacks at the somatic cell level in addition to genetic factors (3). This also indicated that individualized treatment should be adopted for heterogeneous patients of different or the same pedigrees.

The typical genotype-phenotype correlation dictates that VHL missense mutations are responsible for type II disease with a high risk of PHEO and that truncating mutations are responsible for type I disease with a low risk of PHEO (24). Deletion of all or part of the VHL gene as well as the nearby gene BRK1 (also known as HSPC300 and C3orf10) leads to retinal HB and $\mathrm{CHB}$ with a low risk of $\mathrm{RCC}$, which is sometimes called type IB VHL disease (25). The results of the present study supported the notion that missense mutations tend result in PHEO. In addition, mutations at different sites are associated with diverse risks of
VHL-associated lesions (22). Patients with VHL that present with missense mutations in the HIF- $\alpha$ binding site (HM) are associated with a lower risk of PHEO and higher risks of CHB and pancreatic tumors or cysts, while missense mutations located at sites other than that of the HIF- $\alpha$ binding site (nHM) are associated with a higher risk of PHEO, which results in better survival time (24). Furthermore, patients with truncating mutations are more likely to develop RCC than those with HM (26). In contrast to the present study, it has been indicated that p.N78S (HM)-associated PHEO alone, p.R161Q (nHM)-associated RCC/PHEO and p.R167Q (nHM)-associated CNS/RCC/PHEO exhibit diverse phenotypes (16). Of note, the pathogenesis of CHB and RCC, upregulated HIF- $\alpha$ expression and consequent overexpression of VEGF and other HIF-associated genes are the primary causative factors of tumor progression (14). In PHEO, HIF- $\alpha$ (particularly HIF-2 $\alpha$ ) dysregulation results in the overexpression of various HIF-inducible genes, including GLUT1 and VEGF, which themselves serve important roles in tumor development (27). However, the development of PHEO appears to be HIF-independent. In patients with the typical IIC phenotype, as exhibited by the F1 family of the current study, mutant pVHL is able to degrade HIFs, and it has been hypothesized that mutations associated with PHEO may induce gain of function through an intact but altered pVHL (28). In addition, Gossage et al (14) reported that mutations in the elongin $\mathrm{C}$ binding domain of $\mathrm{pVHL}$ are associated with PHEO. These binding sites are implicated in the p53-mediated apoptosis of sympathetic neuronal precursor cells, which then go on to form PHEO (14). It is speculated that such large phenotypic variation may result from numerous other factors that are potentially environmental and may affect the phenotypes induced by specific mutations (7).

The current clinical treatment strategy of VHL disease focuses primarily on the treatment of VHL disease-associated CNS-HB, RCC, PHEO and PNETs (19). The average age of CNS-HB at diagnosis is 33 years (range, $7-78$ years) $(8,19)$. For CNS-HB, 90\% presented in multiple forms and the majority occurred in the cerebellum (45-50\%) and spinal cord $(40-45 \%)(8,20)$. Complete excision of CNS-HB based on microsurgical treatment was rare $(<1 \%)$ with recurrence of the tumor $(8,20)$. Mild nerve defects usually recovered within 2 weeks to 6 months after excision (9). Stereotactic radiotherapy is a potential treatment option for patients who cannot tolerate surgery, or where resection of CNS-HB was difficult. The 2- and 15-year remission rates were 91 and 51\%, respectively $(10,11)$. A previous study also demonstrated that a gamma ray single dose of $20 \mathrm{~Gy}$ at the peripheral position and a 40 Gy dose at the central position could effectively treat CNS-HB (12). Consistently, the F3-III2 in this study was only treated by cranial gamma knife radiotherapy and was stable after 5 years of follow-up. These results indicate that radiotherapy may be an alternative treatment method for patients with CNS-HB who refuse to undergo surgical excision, but the long-term effectiveness requires continued observation. Retinal HB can occur in $~ 50 \%$ of patients with VHL (10). The mean age at diagnosis is 25 years (range, 1-68 years) and the disease is characterized by multiple and bilateral tumors, with sizes varying from $<1$ to several optic discs in diameter (29). In general, small lesions can be treated with greater success and fewer complications compared with those that are larger (30). 
The majority of peripheral retinal HB cases can be treated with laser photocoagulation (small peripheral HB), cryotherapy (large HB) or photodynamic therapy (31). However, these treatments cannot be used when the tumor is near the optic nerve, in which case the therapeutic approach is only surveillance, resulting in a high risk of damage to the optic nerve (32). The average age at diagnosis of VHL-RCC is 39 years (range, $13-70$ years) $(13,20)$. VHL-RCC is characterized by simultaneous or heterogeneous bilateral tumors that are multifocal and low grade with a slow progression $(13,20)$. The metastasis rate of VHL-RCC is very low when tumors are $<3 \mathrm{~cm}$ and physical therapy, such as waiting for observation or radiofrequency ablation, may be used in clinical treatment $(13,22)$. Nephron-sparing surgery should be performed if the tumor is $>3 \mathrm{~cm}$, which should effectively decrease renal insufficiency despite the high recurrence rate of localized neoplasms (13).

In the present study, the diagnostic ages of 3 patients with VHL-RCC (F2-II1, F2-II2 and F3-II1) were 33, 32 and 66 years, respectively. F2-II2 underwent radical resection due to multiple and large tumors of the right kidney. The other two cases underwent unilateral single tumor enucleation (similar to biopsy for definite pathology), all of which were Fuhrman grade II and RCC was relatively stable after follow-up. The average age at diagnosis of VHL-PHEO was 27 years (range, 2.75-58.00 years) $(33,34)$. Of the VHL-PHEO cases, $90 \%$ of tumors were located in the adrenal gland, and $20-50 \%$ of VHL-PHEO were bilateral (33). Malignant VHL-PHEO was rare (1-5\%) (33). VHL-PHEO can be asymptomatic at an early stage and often secretes large quantities of norepinephrine, which differs from MEN2-PHEO, where large quantities of adrenaline are secreted (2,33-35). PHEO excision or partial adrenalectomy decreases the risk of or avoids adrenal cortical insufficiency or crisis (36). It is worth noting that, $50 \%$ of patients developed a second PHEO within 30 years after initial diagnosis $(2,36,37)$. In the present study, the average diagnostic age of 5 patients with VHL-PHEO was 34.6 years. There were 3 patients (F1-II6, F2-II1 and F3-II1) with unilateral PHEO and two patients (F1-III2 and F1-III6) with bilateral PHEO, including one patient (F1-III2) with first-time diagnosis of unilateral PHEO and contralateral PHEO occurring 8 years after surgery. Patients received PHEO resection, after which adrenal function was normal. A previous study determined that the average diagnostic age of PNETs was 35 years (range, 10-75 years). PNETs were asymptomatic and grew slowly. Of the PNETs, $80-93 \%$ were $<3.0 \mathrm{~cm}$. The phenomena of tumor $>3.0 \mathrm{~cm}$, doubling time $<500$ days and codon 161 and 167 mutations at exon 3 were potentially malignant signs $(3,38,39)$. In the present study, one patient (F3-II1, 66 years old) with PNETs required close monitoring for the possibility of malignancy, although no definite signs or symptoms were identified via clinical imaging. VHL-associated renal and pancreatic cysts and epididymis/broad ligament cystadenoma of the uterus is usually asymptomatic and often not treated clinically. The average diagnostic age of ELSTs is 22 years (range, 12-50 years). Of the ELSTs recorded in a previous study, $30 \%$ were bilateral and the majority were benign. Clinical symptoms of ELSTs include hearing loss (84-100\%), tinnitus (73-77\%), dizziness $(62-68 \%)$ and facial paralysis $(8 \%)$. Surgical resection of the tumor is the primary treatment method for ELSTs $(2,3,9)$. In the present study, 10 patients with
VHL disease were not associated with ELSTs and it was speculated that the phenomenon may be associated with the small sample size of the family and patients. In addition, two asymptomatic VHL gene carriers (F2-III1, 5 years of age, p.N78S; F3-IV2, 21 years of age, p.R167Q) were identified via gene testing. Early diagnosis and genetic counseling, regular cancer screening and surveillance are therefore helpful for disease management and for the improvement of prognosis (40).

In conclusion, VHL is a complex disease that can be easily misdiagnosed. Future studies should aim to improve the understanding and attention to molecular diagnosis and management based on VHL gene detection, which may be helpful for early diagnosis and standardization of treatment, improvement of patient prognosis and quality of life, and for the decrease in clinical risk of VHL disease.

\section{Acknowledgements}

Not applicable.

\section{Funding}

No funding was received.

\section{Availability of data and materials}

The datasets used and/or analysed during the current study are available from the corresponding author on reasonable request.

\section{Authors' contributions}

HZ designed the study. GL collected the clinical data, performed the experiments and wrote the manuscript. YZ and $\mathrm{ZZ}$ collected clinical data. All authors read and approved the final manuscript.

\section{Ethics approval and consent to participate}

The present study was approved by the Ethics Committee of Lishui People's Hospital, The Second Affiliated Hospital of Zhejiang University School of Medicine and the First People's Hospital of Wenling City. Written informed consent was obtained from all participants.

\section{Patient consent for publication}

Patients provided their written consent for the publication of these images.

\section{Competing interests}

The authors declare that they have no competing interests.

\section{References}

1. Online Mendelian Inheritance in Man (OMIM) 193300. BioPortal: Nov 18, 2019 (https://www.uniprot.org/database/DB-0062).

2. Ganeshan D, Menias CO, Pickhardt PJ, Sandrasegaran K, Lubner MG, Ramalingam $P$ and Bhalla S: Tumors in von Hippel-Lindau syndrome: From head to toe-comprehensive state-of-the-art review. Radiographics 38: 849-866, 2018. 
3. Chittiboina P and Lonser RR: von Hippel-Lindau disease. In: Handbook of Clinical Neurology. Elsevier, pp139-156, 2015.

4. Aronoff L, Malkin D, van Engelen K, Gallinger B, Wasserman J, Kim RH, Villani A, Meyn MS and Druker H: Evidence for genetic anticipation in vonHippel-Lindau syndrome. J Med Genet 55: 395-402, 2018.

5. Kruizinga RC, Sluiter WJ, de Vries EG, Zonnenberg BA, Lips CJ, van der Horst-Schrivers AN, Walenkamp AM and Links TP: Calculating optimal surveillance for detection of von Hippel-Lindau-related manifestations. Endocr Relat Cancer 21: 63-71, 2013

6. Palmer LS and Linehan WM: Editorial comment. J Urol 183 2351-2351, 2010

7. Iida K, Okimura Y, Takahashi K, Inomata S, Iguchi G, Kaji H and Chihara K: A variety of phenotype with R161Q germline mutation of the von Hippel-Lindau tumor suppressor gene in Japanese kindred. Int J Mol Med 13: 401-404, 2004.

8. Gong K, Zhang N, Zhang $\mathrm{K}$ and $\mathrm{Na} \mathrm{Y}$ : The relationship of erythropoietin overexpression with von Hippel-Lindau tumour suppressor gene mutations between hypoxia-inducible factor-1a and $-2 \alpha$ in sporadic clear cell renal carcinoma. Int J Mol Med 26 907-912, 2010

9. David Dornbos 3III, Kim HJ, Butman JA and Lonser RR: Review of the neurological implications of von Hippel-Lindau disease. JAMA Neurol 75: 620-627, 2018.

10. Asthagiri AR, Mehta GU, Zach L, Li X, Butman JA Camphausen KA and Lonser RR: Prospective evaluation of radiosurgery for hemangioblastomas in von Hippel-Lindau disease. Neuro Oncol 12: 80-86, 2010

11. Lonser RR, Butman JA, Huntoon K, Asthagiri AR, Wu T, Bakhtian KD, Chew EY, Zhuang Z, Linehan WM and Oldfield EH: Prospective natural history study of central nervous system hemangioblastomas in von Hippel-Lindau disease. J Neurosurg 120: 1055-1062, 2014

12. Nambu S, Otani R, Higuchi F, Uzuka T, Matsuda H, Kim P and Ueki K: Histology of hemangioblastoma treated with stereotactic radiosurgery confirms its effectiveness. J Clin Neurosci 51 : 43-45, 2018

13. Kim E and Zschiedrich S: Renal cell carcinoma in von Hippel-Lindau disease-from tumor genetics to novel therapeutic strategies. Front Pediatr 6: 16, 2018.

14. Gossage L, Eisen T and Maher ER: VHL, the story of a tumour suppressor gene. Nat Rev Cancer 15: 55-64, 2015.

15. Liu SJ, Wang JY, Peng SH, Li T, Ning XH, Hong BA, Liu JY, Wu PJ, Zhou BW, Zhou JC, et al: Genotype and phenotype correlation in von Hippel-Lindau disease based on alteration of the HIF- $\alpha$ binding site in VHL protein. Genetics in Medicine Official Journal of the American College of Medical Genetics, 2018.

16. Liu Q, Yuan G, Tong D, Liu G, Yi Y, Zhang J, Zhang Y, Wang LA, Wang L, Zhang D, et al: Novel genotype-phenotype correlations in five Chinese families with Von Hippel-Lindau disease. Endocr Connect 7: 870-878, 2018.

17. Smith ZL, Pietzak EJ, Meise CK, Van Arsdalen K, Wein AJ, Malkowicz SB and Guzzo TJ: Simplification of the Fuhrman grading system for renal cell carcinoma. Can J Urol 22: 8069-8073, 2015.

18. Ben-Skowronek I and Kozaczuk S: Von Hippel-Lindau Syndrome. Horm Res Paediatr 84: 145-152, 2015.

19. Chou A, Toon C, Pickett J and Gill AJ: von Hippel-Lindau syndrome. Front Horm Res 41: 30-49, 2013.

20. Arjumand W and Sultana S: Role of VHL gene mutation in human renal cell carcinoma. Tumour Biol 33: 9-16, 2012

21. Tarade D and Ohh M: The HIF and other quandaries in VHL disease. Oncogene 37: 139-147, 2018.

22. Ordóñez-Navadijo Á, Fuertes-Yebra E, Acosta-Iborra B, Balsa E, Elorza A, Aragonés J and Landazuri MO: Mutant versions of von Hippel-Lindau (VHL) can protect HIF1 $\alpha$ from SART1-mediated degradation in clear-cell renal cell carcinoma. Oncogene 35: 587-594, 2016

23. Clifford SC, Cockman ME, Smallwood AC, Mole DR, Woodward ER, Maxwell PH, Ratcliffe PJ and Maher ER: Contrasting effects on HIF-1alpha regulation by disease-causing pVHL mutations correlate with patterns of tumourigenesis in von Hippel-Lindau disease. Hum Mol Genet 10: 1029-1038, 2001.
24. Shuin T, Ashida S, Yao M and Kanno H: Von Hippel-Lindau disease. Nihon Rinsho 58: 1448-1454, 2000 (In Japanese).

25. Nordstrom-O'Brien M, van der Luijt RB, van Rooijen E, van den Ouweland AM, Majoor-Krakauer DF, Lolkema MP, van Brussel A, Voest EE and Giles RH: Genetic analysis of von Hippel-Lindau disease. Hum Mutat 31: 521-537, 2010.

26. Liu SJ, Wang JY, Peng SH, Li T, Ning XH, Hong BA, Liu JY, Wu PJ, Zhou BW, Zhou JC, et al: Genotype and phenotype correlation in von Hippel-Lindau disease based on alteration of the HIF-alpha binding site in VHL protein. Genet Med 20: 1266-1273, 2018.

27. Young RM and Simon MC: Untuning the tumor metabolic machine: HIF- $\alpha$ : pro- and antitumorigenic? Nat Med 18: 1024-1025, 2012.

28. Hoffman MA, Ohh M, Yang H, Klco JM, Ivan M and Kaelin WG Jr: von Hippel-Lindau protein mutants linked to type $2 \mathrm{C}$ VHL disease preserve the ability to downregulate HIF. Hum Mol Genet 10: 1019-1027, 2001.

29. Niemelä M, Lemeta S, Sainio M, Rauma S, Pukkala E, Kere J, Böhling T, Laatikainen L, Jääskeläinen J and Summanen P: Hemangioblastomas of the retina: Impact of von Hippel-Lindau disease. Invest Ophthalmol Vis Sci 41: 1909-1915, 2000.

30. González Escobar AB, Morillo Sánchez MJ and García-Campos JM: Von Hippel-Lindau disease: Family study. Arch Soc Esp Oftalmol 87: 368-372, 2012 (In Spanish).

31. Richard S, Gardie B, Couvé S and Gad S: Von Hippel-Lindau: How a rare disease illuminates cancer biology. Semin Cancer Biol 23: 26-37, 2013.

32. Lefevre A, Mathis T, Denis P and Kodjikian L: Retinal hemangioblastoma: Treatment strategy and long-term follow-up in a retrospective cohort. J Fr Ophtalmol 41: 164-169, 2018 (In French).

33. Woodward ER and Maher ER: Von Hippel-Lindau disease and endocrine tumour susceptibility. Endocr Relat Cancer 13: 415-425, 2006.

34. Sovinz P, Urban C, Uhrig S, Stepan V, Lackner H, Schwinger W, Benesch M, Moser A, Spuller E and Speicher MR: Pheochromocytoma in a 2.75-year-old-girl with a germline von Hippel-Lindau mutation Q164R. Am J Med Genet A 152A: 1752-1755, 2010.

35. Colvin A, Saltzman AF, Walker J, Bruny J and Cost NG: Metastatic pheochromocytoma in an asymptomatic 12-Year-Old with von Hippel-Lindau disease. Urology 119: 140-142, 2018.

36. Petr EJ and Else T: Genetic predisposition to endocrine tumors: diagnosis, surveillance and challenges in care. Semin Oncol 43: 582-590, 2016.

37. Lenders JW, Duh Q-Y, Eisenhofer G, Gimenez-Roqueplo AP, Grebe SK, Murad MH, Naruse M, Pacak K and Young WF Jr; Endocrine Society: Pheochromocytoma and paraganglioma: An endocrine society clinical practice guideline. J Clin Endocrinol Metab 99: 1915-1942, 2014

38. Tirosh A, Sadowski SM, Linehan WM, Libutti SK, Patel D, Nilubol N and Kebebew E: Association of VHL genotype with pancreatic neuroendocrine tumor phenotype in patients with von Hippel-Lindau disease. JAMA Oncol 4: 124-126, 2018.

39. Krauss T, Ferrara AM, Links TP, Wellner U, Bancos I, Kvachenyuk A, Villar Gómez de Las Heras K, Yukina MY, Petrov R, Bullivant G, et al: Preventive medicine of von Hippel-Lindau disease-associated pancreatic neuroendocrine tumors. Endocr Relat Cancer 25: 783-793, 2018.

40. Wang J-Y, Peng S-H, Li T, Ning XH, Liu SJ, Hong BA, Liu JY, Wu PJ, Zhou BW, Zhou JC, et al: Risk factors for survival in patients with von Hippel-Lindau disease. J Med Genet 55: 322-328, 2018.

This work is licensed under a Creative Commons Attribution-NonCommercial-NoDerivatives 4.0 International (CC BY-NC-ND 4.0) License. 